Erratum

\title{
Erratum to "Impact of Yoga and Meditation on Cellular Aging in Apparently Healthy Individuals: A Prospective, Open-Label Single-Arm Exploratory Study"
}

\author{
Madhuri Tolahunase, ${ }^{1}$ Rajesh Sagar, ${ }^{2}$ and Rima Dada ${ }^{1}$ \\ ${ }^{1}$ Lab for Molecular Reproduction and Genetics, Department of Anatomy, All India Institute of Medical Sciences (AIIMS), \\ New Delhi, India \\ ${ }^{2}$ Department of Psychiatry, All India Institute of Medical Sciences (AIIMS), New Delhi, India
}

Correspondence should be addressed to Rima Dada; rima_dada@rediffmail.com

Received 9 August 2017; Accepted 28 August 2017; Published 24 September 2017

Copyright (C) 2017 Madhuri Tolahunase et al. This is an open access article distributed under the Creative Commons Attribution License, which permits unrestricted use, distribution, and reproduction in any medium, provided the original work is properly cited.

In the article titled "Impact of Yoga and Meditation on Cellular Aging in Apparently Healthy Individuals: A Prospective, Open-Label Single-Arm Exploratory Study" [1], there were a number of language errors, due to publisher error. The corrected version of the article is shown below:

\begin{abstract}
This study was designed to explore the impact of a yoga- and meditation-based lifestyle intervention (YMLI) on cellular aging in apparently healthy individuals. During this 12 -week prospective, open-label, single-arm exploratory study, 96 apparently healthy individuals were enrolled to receive YMLI. The primary endpoints were assessment of the change in levels of cardinal biomarkers of cellular aging in blood from baseline to week 12, which included DNA damage marker 8 -hydroxy- $2^{\prime}$-deoxyguanosine $(8-\mathrm{OH} 2 \mathrm{dG})$, oxidative stress markers reactive oxygen species (ROS), and total antioxidant capacity (TAC), and telomere attrition markers telomere length and telomerase activity. The secondary endpoints were assessment of metabotrophic blood biomarkers associated with cellular aging, which included cortisol, $\beta$-endorphin, IL6 , BDNF, and sirtuin-1. After 12 weeks of YMLI, there were significant improvements in both the cardinal biomarkers of cellular aging and the metabotrophic biomarkers influencing cellular aging, compared to baseline values. The mean levels of 8-OH2dG, ROS, cortisol, and IL-6 were significantly lower
\end{abstract}

and mean levels of TAC, telomerase activity, $\beta$-endorphin, BDNF, and sirtuin-1 were significantly increased (all values $p<0.05)$ post-YMLI. The mean level of telomere length was increased but the finding was not significant $(p=0.069)$. YMLI significantly reduced the rate of cellular aging in an apparently healthy population.

\section{Introduction}

In the last decade there has been a significant increase in complex lifestyle diseases like depression, diabetes mellitus (DM), cardiovascular diseases (CVD), cancer, and infertility. These diseases are strongly associated with accelerated cellular aging $[1,2]$ and have become the bane of modern society [3-5]. Within a homogeneous sample of an apparently healthy adult population, biomarkers have been defined recently [6] to characterize the complex processes of an accelerated aging phenomenon. Although we do not have any gold standard biomarker to monitor healthy aging, based on the current knowledge of putative biomarkers, the cardinal biomarkers of cellular aging and metabotophic biomarkers that can influence them have become the focus of the latest translational research to develop interventions to prevent chronic lifestyle diseases.

The cardinal biomarkers of cellular aging include DNA damage, telomere length attrition, and oxidative stress (OS) [7]. DNA damage causes genomic instability, which is 
responsible for cellular dysfunction in the pathogenesis of lifestyle diseases [8-10]. OS is the most important cause of DNA damage. Although many different oxidative DNA damage (ODD) products have been identified, 8-OH2dG (8hydroxy-2'-deoxyguanosine), a highly mutagenic oxidative DNA adduct, has been the subject of intensive study and is a definitive biomarker of DNA damage [11]. Telomere attrition is due to altered telomere metabolism involving a decrease in telomerase enzyme activity and an increase in OS. It contributes to genomic instability and is associated with aging and lifestyle diseases [12].

Oxidative stress, an imbalance between prooxidants and antioxidant defense mechanisms, becomes pathological at both extremes of the physiological range needed for normal cellular functions. It is involved in the pathogenesis of complex lifestyle and chronic diseases [13], including depression [14], obesity [15], and infertility [16, 17], the leading public health problems.

Several metabotrophic blood biomarkers influencing cellular aging include biomarkers of stress and inflammatory response, neuroplasticity, and longevity. Sustained stress responses due to chronic stress stimuli cause constantly increased cortisol levels [18], which lead to systemic tissue abnormalities like increased adiposity and neurodegeneration. The level of stress responsiveness (cortisol levels) can be a biomarker for predicting susceptibility to lifestyle diseases [19]. Accelerated aging is characterized by a chronic, low-grade inflammation ("inflammaging"). Inflammaging is a highly significant risk factor for most chronic lifestyle diseases [20] and is a potential modifiable target [21]. IL-6 is the most prominent cytokine in inflammaging and is both a marker of inflammatory status and a hallmark of chronic morbidity [22]. Impaired neuroplasticity due to accelerated aging can have negative influence across the entire lifespan [23]. BDNF is a major regulator of neuroplasticity [24], which may be increased in specific regions of the brain by various interventions [25]. Health span and longevity are influenced by several factors. Sirtuin-1 (SIRT1), a histone deacetylase (HDAC), is prominent among them and recently has become a target for various interventions [26]. It systemically influences nutrition and energy metabolism and centrally has a role in circadian rhythm, survival against stress [27] and neuronal plasticity [28].

A variety of interventions have been studied $[29,30]$ to determine their influence on preventing lifestyle diseases and promoting health and longevity. They include treatments targeting specific hallmarks of aging, namely, physical exercise [31], nutrition, caloric restriction [32], and antioxidants [33]. However, no single intervention is shown to be an effective preventive and therapeutic strategy for modern complex lifestyle diseases and to provide comprehensive benefits for delaying or reversing accelerated aging. Therefore, further research is needed to find optimum interventions for population at risk of lifestyle diseases. Yoga is an emerging integrative health discipline, which can positively modulate mind and body [34] and has been shown to improve the clinical profile of patients with various pathologies [35] including depression, obesity, hypertension, asthma, type II diabetes, and cancer. However, recent reviews on yoga suggest that potential underlying mechanisms need to be further explored [36]. Studies on biomarkers of disease and health in yoga-based interventions are limited and they have only highlighted diabetic and lipid profiles [37,38], stress and inflammatory markers [39, 40] and neuroimaging correlates [41], in populations with specific medical conditions. Evidence is lacking regarding the efficacy of yoga lasting a short duration of 3 to 12 weeks in improving the biomarkers of cellular aging in apparently healthy people. Thus, the present study was designed to evaluate the impact of a yoga- and meditationbased lifestyle intervention (YMLI) on cellular aging and longevity by analyzing cardinal and metabotrophic biomarkers in the peripheral blood of apparently healthy subjects.

\section{Materials and Methods}

2.1. Study Design and Participants. Ninety-six apparently healthy people were enrolled in this 12 -week prospective, open-label, single-arm exploratory study, from August 2015 to May 2016, designed to explore the impact of YMLI on cellular aging. The key inclusion criteria were male or female aged 30-65 years and leading an unhealthy modern lifestyle. The key exclusion criteria were inability to perform the yogic exercises due to any physical challenges and those with recent changes in lifestyle during the last 3 months. The study was initiated after ethical clearance (ESC/T-370/22-07-2015) and the registration of the trial with the Clinical Trial Registry of India (CTRI REF/2014/09/007532).

\subsection{Procedure}

2.2.1. Yoga- and Meditation-Based Lifestyle Intervention (YMLI). Eligible subjects were enrolled in the study after screening and baseline characteristics were recorded. Participants underwent a 12-week pretested YMLI program comprising theory and practice sessions $[42,43]$. YMLI is designed to be an integrative health strategy incorporating the classic components of yoga including asanas (physical postures), pranayama (breathing exercises), and dhayna (meditation), which are derived from a mix of Hatha yoga and Raja yoga. The YMLI for the current study was suitably modified for apparently healthy subjects. YMLI program included sessions five days per week for 12 weeks. For the first two weeks, the sessions were held at the integrated health clinic (IHC), AIIMS, New Delhi, and taught by registered, specialized yoga instructors (educational qualifications include Bachelor of Naturopathy and Yoga Sciences and P.G. Diploma in Yoga Therapy). The remaining 10 weeks were home based. Monitoring of compliance of the home-based YMLI was through maintenance of a diary and telephonic contact. The details of the activities in a day during the YMLI program are given in Table 1. Each session in YMLI included a set of asanas, pranayama, and dhayna for approximately $90 \mathrm{~min}-$ utes. This was followed by an interactive lecture (only during the first two weeks of YMLI at IHC) on lifestyle, lifestyle diseases, and importance of their prevention for 30 minutes.

2.2.2. Laboratory Procedures. During this 12 -week study the participants were evaluated for various biomarkers on day 
TABLE 1: Details of activities in a day of Yoga- and Meditation-based Lifestyle Intervention (YMLI) program.

\begin{tabular}{|c|c|c|c|c|}
\hline S. No. & \multicolumn{2}{|c|}{ Practice to be done } & & \multirow{2}{*}{$\begin{array}{c}\text { Duration } \\
5 \mathrm{~min}\end{array}$} \\
\hline$(1)$ & Session preparation instructions & & & \\
\hline \multirow{2}{*}{$(2)$} & Prayer & & & $3 \mathrm{~min}$ \\
\hline & Loosening practices (warm-up) & & & $5 \mathrm{~min}$ \\
\hline \multirow{12}{*}{ (3) } & \multirow{12}{*}{ Asanas (Postures) } & \multirow{3}{*}{ Supine } & Shavasana & $2 \mathrm{~min}$ \\
\hline & & & Uttanpadasana & $2 \mathrm{~min}$ \\
\hline & & & Pawanmuktasana & $2 \min$ \\
\hline & & \multirow{3}{*}{ Prone } & Makarasana & $2 \min$ \\
\hline & & & Bhujangasana & $2 \min$ \\
\hline & & & Salabhasana & $2 \min$ \\
\hline & & \multirow{3}{*}{ Sitting } & Vakrasana & $2 \mathrm{~min}$ \\
\hline & & & Ardha-Matsyendrasana & $2 \min$ \\
\hline & & & Vajrasana & $2 \mathrm{~min}$ \\
\hline & & \multirow{3}{*}{ Standing } & Tadasana & $2 \mathrm{~min}$ \\
\hline & & & Vrikshasana & $2 \min$ \\
\hline & & & Ardhachakrasana & $2 \min$ \\
\hline$(4)$ & Relaxation & & Shavasana & $5 \mathrm{~min}$ \\
\hline \multirow{5}{*}{ (5) } & \multirow{5}{*}{ Pranayama (Breathing Exercises) } & & Nadishodhana & \multirow{5}{*}{$20 \mathrm{~min}$} \\
\hline & & & Bhramri & \\
\hline & & & Shitkari & \\
\hline & & & Shitali & \\
\hline & & & Brahmamudra & \\
\hline$(6)$ & Aumkar recitation & & & $3 \min$ \\
\hline$(7)$ & Dhyana (Meditation) & & & $20 \mathrm{~min}$ \\
\hline$(8)$ & Shanti mantra & & & $5 \mathrm{~min}$ \\
\hline$(9)$ & $\begin{array}{c}\text { Interactive session (first } 2 \text { weeks only at } \\
\text { Integrated Health Clinic, AIIMS, New } \\
\text { Delhi) }\end{array}$ & & & $30 \mathrm{~min}$ \\
\hline Total & & & & $120 \mathrm{~min}$ \\
\hline
\end{tabular}

0 and week 12. Fasting venous blood samples $(5 \mathrm{~mL})$ were collected and divided into two parts. One part was allowed to clot and the serum was separated within 30 minutes and the other part was transferred to heparinized/EDTA vials and was centrifuged at $2000 \mathrm{~g}$ for 15 minutes at $4^{\circ} \mathrm{C}$. Both serum and plasma were stored at $-80^{\circ} \mathrm{C}$ until analyzed. ROS detection was done by chemiluminescence assay (Berthold detection luminometer, USA). Peripheral blood leukocyte telomere length was measured by qPCR and telomerase activity was determined by using a telomerase assay kit (Roche, Switzerland), as per the manufacturer's protocol. 8-OH2dG was estimated in white blood cell DNA (Cayman's EIA kit). ELISA kits were used for levels of TAC (Cayman Chemical, Ann Arbor, USA), cortisol (DRG Diagnostic, Germany), $\beta$-endorphin (Phoenix Pharmaceuticals, Inc.), IL-6 (GenProbe, Diaclone Diagnostic, France), BDNF (Raybiotech, Inc), and sirtuin-1 (Quayee Bio-Technology). Quality control assays for biomarkers and validation were performed.

2.2.3. Endpoints. The primary endpoint was to assess the change in levels of cardinal biomarkers of cellular aging from baseline to week 12. The biomarkers included the following:
8-OH2dG, ROS, and TAC (markers of OS and ODD) as well as telomere length and telomerase activity (telomere attrition markers). The secondary endpoints were assessment of metabotrophic blood biomarkers associated with cellular aging, which included cortisol, $\beta$-endorphin, IL-6, BDNF, and sirtuin-1 from baseline to week 12 .

2.3. Statistical Analysis. Data were analyzed using SPSS 20 (IBM Corp, Armonk, NY). Descriptive statistics are reported as means and standard deviations. Changes in outcome variables were analyzed using paired-samples $t$-tests. Exploratory analysis included comparisons for within gender subgroups using paired-sample $t$-tests. Significance was accepted at $p<$ 0.05 .

\section{Results}

The flow diagram of participant details is provided in Figure 1. Of 96 subjects, 94 subjects were assessed for impact analysis. Two subjects were excluded from the analysis due to poor compliance to the program. Baseline sociodemographic characteristics are shown in Table 2. 


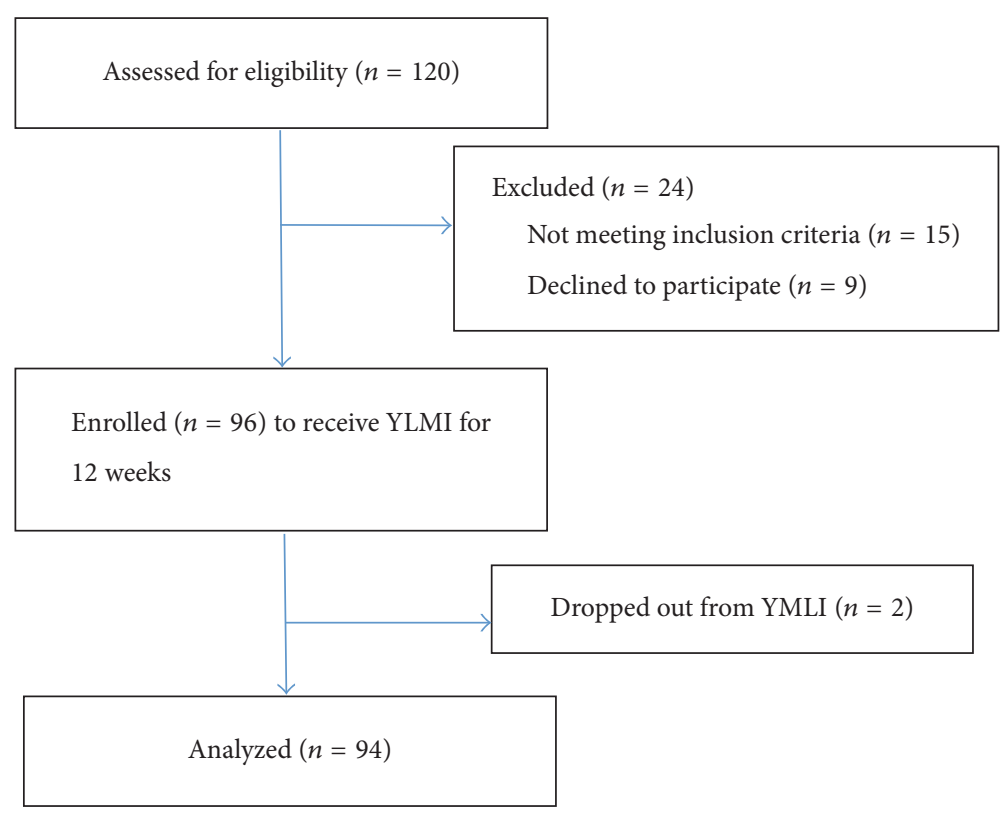

FIgURE 1: Flow diagram of study participation.

TABLE 2: Sociodemographic characteristics of participants.

\begin{tabular}{|c|c|}
\hline Variable & Values \\
\hline Age (years) & $40.26(10.13)$ \\
\hline \multicolumn{2}{|l|}{ Sex } \\
\hline Female & $52(55.32)$ \\
\hline Male & $42(44.68)$ \\
\hline \multicolumn{2}{|c|}{ Socioeconomic status } \\
\hline \multicolumn{2}{|c|}{ Kuppuswamy socioeconomic status scale } \\
\hline Education & $4.82(1.24)$ \\
\hline Occupation & $5.26(2.38)$ \\
\hline Income & $8.60(2.74)$ \\
\hline Total & $18.68(7.34)$ \\
\hline $\operatorname{BMI}\left(\mathrm{kg} / \mathrm{m}^{2}\right)$ & $26.30(3.40)$ \\
\hline
\end{tabular}

Data were described as frequency (\%) for sex and mean (SD) for others.

After 12 weeks of YMLI, there was significant improvement in both cardinal and metabotrophic biomarkers of cellular aging compared to baseline values (Table 3). The mean levels of 8-OH2dG and ROS were significantly lower and mean levels of TAC and telomerase activity were significantly increased (all values $p<0.05$ ). The mean level of telomere length was increased, but this finding was not significant $(p=$ 0.069 ). The mean levels of cortisol and IL- 6 were significantly lower and mean levels of $\beta$-endorphin, BDNF, and sirtuin- 1 were significantly increased (all values $p<0.05$ ).

A few differences were noted in the gender subgroup analysis. Only the male subgroup showed a significant decrease in the levels of IL- 6 and a more marked reduction in cortisol levels (males $p=0.001$; females $p=0.036$ ). After 12 weeks of YMLI, we also noted significantly reduced BMI in the study population $(p<0.01)$.

\section{Discussion}

The results of this study highlight the positive impact of YMLI on biomarkers of cellular aging and in promoting cellular longevity through changes in both cardinal and metabotrophic biomarkers. The findings suggest that the impact is mediated through improvement in genomic stability, telomere metabolism, and balance of cellular oxidative stress, well-regulated stress and inflammatory responses, and increase in neuroplasticity and nutrition sensing.

Genomic stability is central to cellular longevity and disease-free youthful healthy life and the findings from our study suggest the reduction of genomic instability (decreased levels of 8-OH2dG) by YMLI. Unhealthy social habits (smoking, excess alcohol intake, etc.), sedentary lifestyle, exposure to environmental pollutants, and intake of processed and nutritionally depleted food have taken a toll on human health, with the onset of lifestyle diseases at a much younger age [3-5]. These environmental and lifestyle factors are responsible for genomic instability [10]. DNA damage to both the mitochondrial and nuclear genome from endogenous as well as exogenous insults results in accumulation of genetic aberrations and genome hypermutability [8-10].

This is mainly due to aberrant DNA damage response (DDR) pathway, which is essential for DNA repair and for monitoring genomic integrity. Deficient DNA repair triggers systemic effects to promote pathological aging [10]. The reduction of DNA damage by YMLI suggests the potential of yoga in activating the DDR pathway to repair genomic damage and improve genomic stability, and changes in metabotrophic factors seen in the study may be associated with these benefits.

Maintaining telomere length through regulation of telomere metabolism contributes to genomic stability, and the reduction in telomere attrition (increase in telomere 
TABLE 3: Change in outcomes in apparently healthy sedentary subjects participating in a Yoga- and Meditation-based lifestyle intervention $(n=94)$.

\begin{tabular}{|c|c|c|c|c|c|}
\hline Characteristics & Baseline & $12 \mathrm{wks}$ & $\begin{array}{c}\text { Change from baseline to } \\
12 \mathrm{wks} \\
\text { (diff. } 95 \% \text { C.I.) }\end{array}$ & Effect size $^{*}$ & $p$ value \\
\hline \multicolumn{6}{|c|}{$\begin{array}{l}\text { Primary endpoints: cardinal biomarkers } \\
\text { of cellular aging }\end{array}$} \\
\hline \multicolumn{6}{|l|}{ Oxidative stress } \\
\hline ROS (RLU/min $/ 10^{4}$ neutrophils) & $1215.069 \pm 88$ & $1020.81 \pm 79$ & $194.3(164,224.5)$ & 0.7 & $<0.0001$ \\
\hline TAC (mmol Trolox equiv/L) & $5.94 \pm 1.52$ & $7.4 \pm 2.1$ & $-1.16(-1.9,-0.41)$ & 0.4 & $<0.001$ \\
\hline \multicolumn{6}{|l|}{ DNA damage } \\
\hline $8 \mathrm{OH} 2 \mathrm{dG}(\mathrm{pg} / \mathrm{mL})$ & $1026.23 \pm 630$ & $790.98 \pm 400$ & $235.3(72.73,397.8)$ & 0.22 & $<0.01$ \\
\hline \multicolumn{6}{|l|}{ Telomere attrition } \\
\hline Telomerase activity (IU/cell) & $1.89 \pm 1.42$ & $2.94 \pm 2.2$ & $-1.05(-1.68,-0.41)$ & 0.3 & $<0.001$ \\
\hline Telomere length (IU/cell) & $2.36 \pm 1.6$ & $2.44 \pm 1.4$ & $-0.08(-0.61,0.45)$ & 0.02 & 0.069 \\
\hline \multicolumn{6}{|l|}{$\begin{array}{l}\text { Secondary endpoints: biomarkers } \\
\text { associated with cellular aging }\end{array}$} \\
\hline Cortisol (ng/mL) & $118.83 \pm 50.50$ & $96.32 \pm 38.6$ & $22.51(7.6,37.42)$ & 0.3 & $<0.01$ \\
\hline Interleukin (IL6) (pg/mL) & $3.16 \pm 2.42$ & $1.94 \pm 2.3$ & $1.22(0.47,1.97)$ & 0.3 & $<0.001$ \\
\hline$\beta$-Endorphins (ng/mL) & $6.2 \pm 3.5$ & $8.2 \pm 4.2$ & $-2(-3.22,-0.77)$ & 0.3 & $<0.001$ \\
\hline $\mathrm{BDNF}(\mathrm{ng} / \mathrm{mL})$ & $19.7 \pm 6.75$ & $37.1 \pm 5.6$ & $-17.4(-19.48,-15.32)$ & 0.7 & $<0.0001$ \\
\hline Sirtuin $(\mathrm{ng} / \mathrm{mL})$ & $26.69 \pm 10.42$ & $40.64 \pm 11.6$ & $-13.95(-23.41,-4.49)$ & 0.5 & $<0.01$ \\
\hline$B M I\left(\mathrm{~kg} / \mathrm{m}^{2}\right)$ & $26.30 \pm 3.40$ & $23.64 \pm 3.55$ & $2.66(0.56,3.12)$ & 0.4 & $<0.01$ \\
\hline
\end{tabular}

${ }^{*}$ Effect size was calculated by dividing change by standard deviation at baseline of the specific outcome and interpreted using Cohen's $d$ (small effect: 0.2 to 0.3 , medium effect: 0.5 , and large effect: 0.8 ).

length and telomerase activity levels) shown by our study after YMLI suggests the potential for yoga in telomere metabolism and cellular longevity. Telomeres, which serve as a biological clock, are highly conserved hexameric repeats and maintaining their length is vital for cellular longevity. Telomerase is an important regulator of telomere length and accurate regulation of its activity, and a correct telomeretelomerase interaction, is important to precisely safeguard telomere length and prevent telomere attrition [44]. ODD is prominent among the factors that can adversely affect telomere length [45]. Rapid telomere attrition due to ODD is associated with senescence and related disease conditions $[46,47]$. Improved telomere metabolism after YMLI seen in the study may contribute to genomic stability. More research is needed to explore the mechanisms of how yoga and meditation can positively modify telomere metabolism.

Our study suggests an improvement in maintenance of balance in cellular oxidative stress (decrease in ROS and increase in TAC) caused by YMLI. Supraphysiological ROS levels are due to endogenous and exogenous factors like smoking, excess alcohol consumption, exposure to electromagnetic radiation, infection, xenobiotic exposure, and psychological stress [48]. Even the levels of ROS below physiological limits are deleterious to normal cellular function and maintaining OS at physiological levels is important for cellular longevity. Increased OS causes damage to all molecules, including damage to DNA and telomeres. It also affects signal transduction and gene transcription by causing genome-wide hypomethylation [49] and thus causes changes in the epigenome. Regulation of cellular oxidative stress within physiological limits after YMLI suggests the potential of this intervention in protecting cells from OSinduced DNA damage and telomere attrition and in reversing epigenetic changes, which are accumulated due to an unhealthy lifestyle and adverse environmental conditions. Other studies [50] support these findings and have shown reduced OS upregulation of telomerase activity and decreased ODD after YMLI. To combat OS people use antioxidants without monitoring ROS levels resulting in reductive stress [51], unlike in YMLI which regulates ROS levels so that no redox-sensitive physiological functions are impaired.

Modern lifestyle and associated psychological stress have complex interactions with habits, environmental conditions, and medical interventions to cause accelerated cellular aging, which adversely affect our mental, physical, and reproductive fitness [3-5]. Improved cellular longevity after YMLI suggests the potential role of yoga in promoting this fitness. While psychological stress has a major effect on the mind, contributing to an increased prevalence of neuropsychiatric disorders including depression, abnormal fat accumulation is a major somatic manifestation contributing to increased prevalence of metabolic syndrome and all the diseases that come under this umbrella including, obesity, DM, and CVD [52]. Other peripheral manifestations of unhealthy modern lifestyle include aging of gonads leading to infertility [53] and recurrent pregnancy loss. Previous studies have demonstrated the clinical benefits of yoga and meditation in all these medical conditions [35]. Dada et al. have shown that 
YMLI can reduce testicular aging and result in significant upregulation in telomerase activity and decline in seminal OS and ODD [8]. The ongoing studies in our laboratory on the impact of yoga and meditation have provided significant evidence for the reversal of cellular aging in subjects prone to accelerated aging due to depression. Microarray (Agilent $8 \times 60 \mathrm{k}$ Microarray kit) and analysis of gene expression preand post-YMLI showed decreased IL6, IL10, and MAP10 and increased IL2 and IL4 $[8,54,55]$. Improved cellular longevity seen in our study after YMLI suggests that changes in both cardinal and metabotrophic biomarkers of cellular aging may be a mechanism for preventing chronic lifestyle diseases. Our study suggests that the changes in metabotrophic factors, which include increase in levels of $\beta$-endorphin, BDNF, and sirtuin-1 and decrease in levels of cortisol and IL-6, and the cellular processes involving them, may have important roles in the reversal of cellular aging and improving cellular longevity after YMLI.

Improvements in stress and inflammatory response in our study after YMLI may be mediated by changes in cortisol, $\beta$-endorphin, IL- 6 , and other factors, with regulation by changes in the brain through the hypothalamic-pituitaryadrenal (HPA) axis. The response may involve regulation of adaptive pathways, including integrated stress response (ISR) [18] that activates eukaryotic translation initiation factor 2 alpha (eIF $2 \alpha$ ), that promote cellular recovery driving signaling toward cell survival and longevity. The response may lead to decreased OS and a reversal of the senescent secretory phenotype of cells, including cells in the brain, adipose tissue, endothelium, and gonads. Changes in secretory phenotype include decreased IL-6 [56], increased BDNF, and sirtuin-1 [57]. These regulated factors may lead to balance in OS and cellular longevity and contribute to tissue revival throughout the body from neuroplasticity in CNS to gonads, vessels, and muscles in the periphery. Secretory phenotype from somatic cells provides regulatory feedback to brain [58], which completes the vicious cycle of regulation between mind and body. Neurodegeneration is associated with the pathogenesis of several neuropsychiatric conditions and neuroplasticity has a central role in their management and for vitality. Increased BDNF, sirtuin-1, and $\beta$-endorphin and decreased cortisol each decrease cellular aging in brain, decrease neurodegeneration and increase neuroplasticity [23]. Increased cellular longevity and increased neuroplasticity may be a mechanism for alteration of gray matter volume in different regions of the cerebral cortex [59], increased mindfulness [60], and several other complex processes [55, 61] involved in reduction of stress and depression after yoga and meditation. Regulated mind-body communications may lead to minimization of subclinical inflammation and activation of nutrition and energy-sensing pathways promoting longevity, where a decrease in IL- 6 and an increase in sirtuin-1 play a prominent role, respectively. Previous studies have demonstrated an increase in sirtuin-1 levels after interventions with caloric restriction [62]. Our study is the first to document an increase in sirtuin-1 levels independent of caloric restriction after practicing yoga. These improved processes may result in delaying onset and slowing down progression of diseases associated with accelerated cellular aging.
The impact of the intervention in both genders was assessed separately since men and women respond differently to day-to-day stress [63]. Interestingly, the gender subgroup analysis showed that reductions in cortisol and IL6 levels were more pronounced in male than in female subjects. No significant gender differences were seen in the other biomarkers. Phase of the menstrual cycle should be taken into consideration, since some biomarker levels are known to vary with different phases of the menstrual cycle $[64,65]$. Our study showed a significant decrease in BMI in apparently healthy subjects, which came into the normal range (23.64 \pm $3.55)$ from a baseline overweight range $(26.30 \pm 3.40)$. While the latest research [66] suggests people with a mean BMI of 27 , who are overweight by the current classification of obesity, are likely to survive longest in a Western population, similar data is not available for the Indian population. Therefore, our findings need to be interpreted cautiously.

Stratification of cases was not done in this study to do a subgroup analysis due to the small sample size.

Lifestyle is an integrated entity, and an intervention like YMLI that has an overall positive influence on our health appears most useful versus changing only one aspect at a time, as is seen by the action of certain drugs. Yoga is holistic and a mind-body medicine and is more beneficial and advantageous than individual interventions like physical exercise, caloric restriction, and antioxidants. The practice of yoga and physical exercise are different entities; the former results in energy conservation with economy of energy expenditure for mental and physical benefits, and the later results in energy expenditure more for physical exertions and metabolic needs, as is evident from a study that showed exercise causes erratic changes in biomarkers and results in OS [67] while meditation brings about uniform biomarker and behavioral changes and improvement in cognition and decrease OS [68]. Therapeutic antioxidants can only decrease ROS rather than regulating it and may paradoxically shorten lifespan [69] due to an imbalance in ROS-mediated immune responses [70]. YMLI regulates ROS rather than simply lowering them by balanced stress-related processes and appropriate gene expression [71]. The only limitation of our study is that it is a single-arm proof-of-concept study and did not include controls. It is important to adopt a lifestyle that slows the decline in health by reversing or delaying accelerated aging due to unhealthy lifestyles. The biomarkers of cellular aging can form the basis for determining the risk of chronic lifestyle diseases and the efficacy and usefulness of interventions to decrease disease risk. Hence, the findings from this study are supportive of YMLI as having significant clinical utility especially in the prevention of and management of complex multifactorial diseases and reducing the rate of functional decline with aging.

\section{Conclusion}

Though we cannot change our biology or chronological age we can definitely reverse/slow down the pace at which we age by adopting YMLI. This is the first study to demonstrate improvement in both cardinal and metabotrophic biomarkers of cellular aging and longevity in an apparently healthy 
population after a yoga- and meditation-based lifestyle intervention. Our health and the rate at which we age entirely depends on our choices. Making yoga and meditation an integral part of our lifestyle may hold the key to delay aging or aging gracefully, prevent the onset of multifactorial complex lifestyle diseases, promote mental, physical, and reproductive health, and prolong youthful healthy life.

\section{Conflicts of Interest}

The authors declare there are no conflicts of interest regarding the publication of this article.

\section{Acknowledgments}

The authors are thankful to Amit Tomar and Sudhir Choudary for yoga instructions and to all the subjects enrolled in the study.

\section{References}

[1] M. Tolahunase, R. Sagar, and R. Dada, "Impact of yoga and meditation on cellular aging in apparently healthy individuals: a prospective, open-label single-arm exploratory study," Oxidative Medicine and Cellular Longevity, vol. 2017, Article ID 7928981, 9 pages, 2017. 


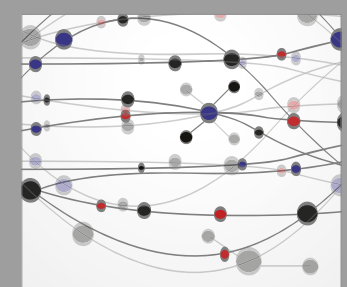

The Scientific World Journal
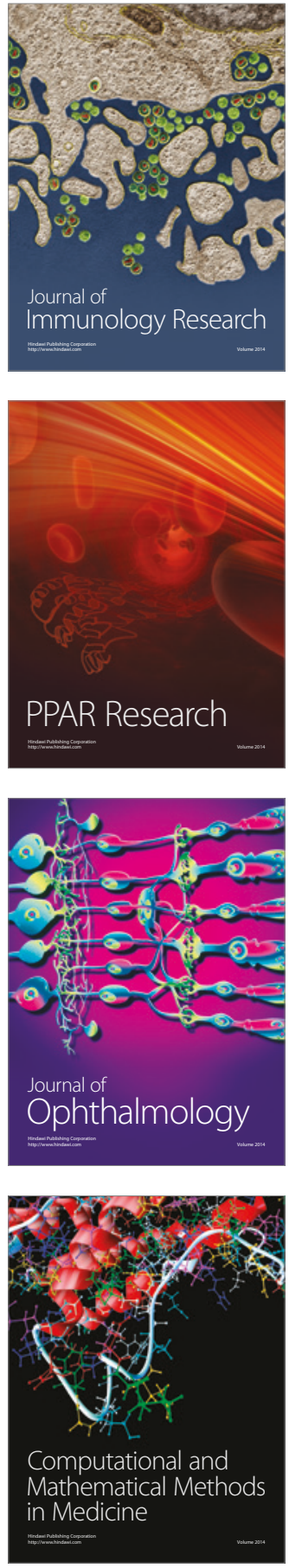

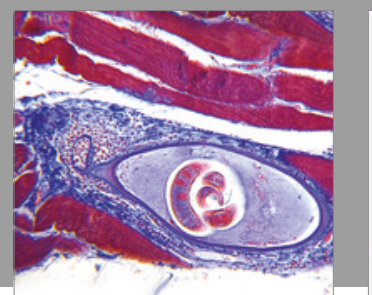

Gastroenterology Research and Practice
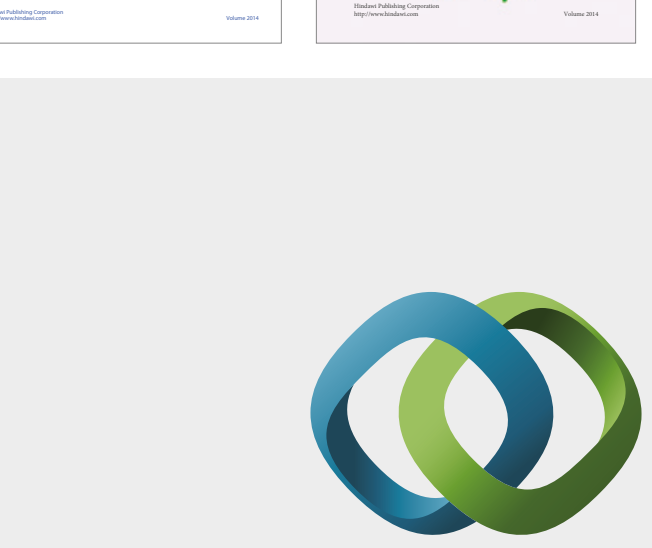

\section{Hindawi}

Submit your manuscripts at

https://www.hindawi.com
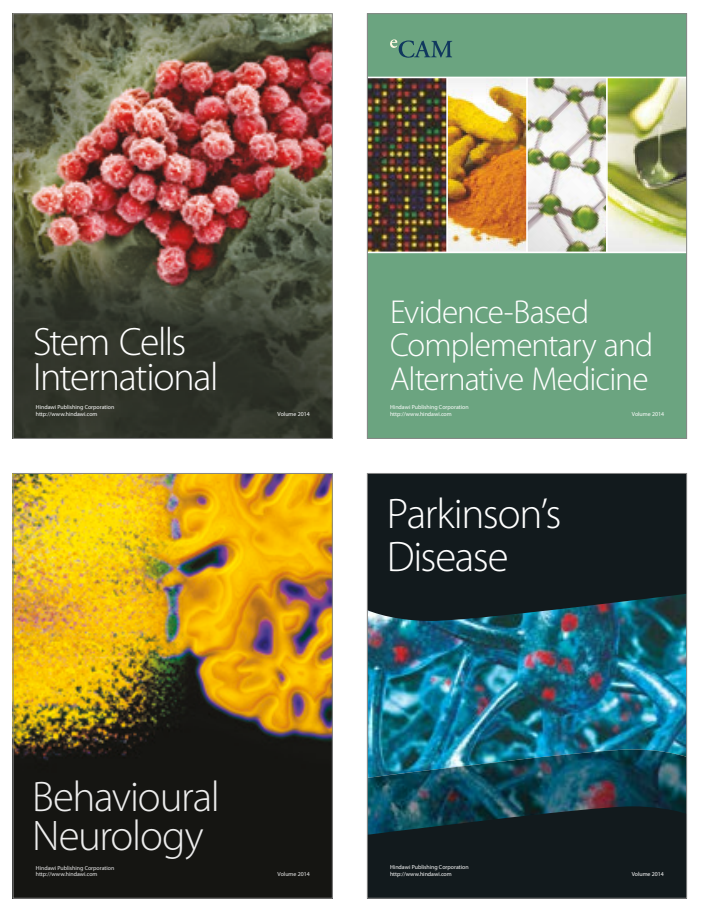
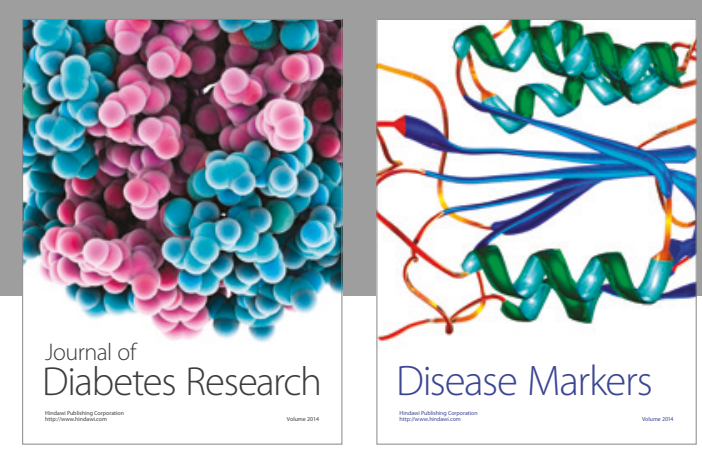

Disease Markers
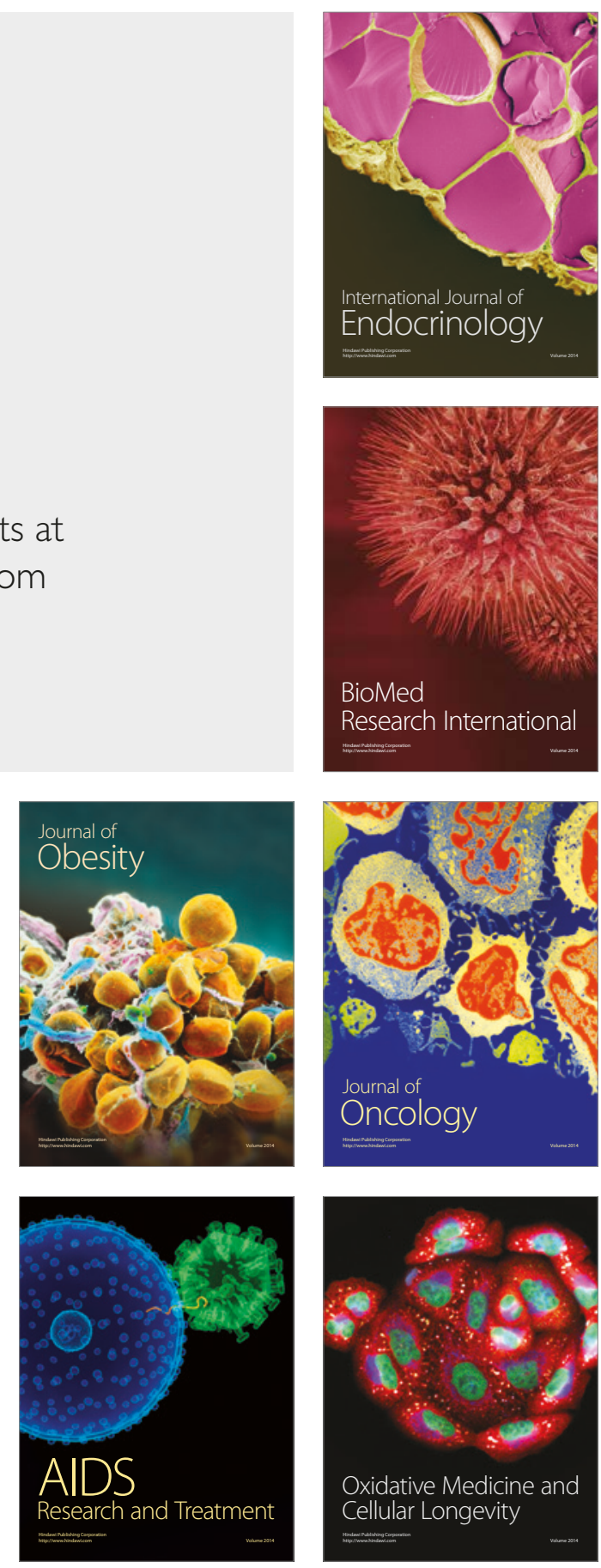